\title{
Detecting Spatial-temporal urban ponding distribution from surveillance videos based on computer vision
}

Xin Hao ( $\sim$ hhaox@mail.dlut.edu.cn )

Dalian University of Technology

Heng Lyu

Purdue University https://orcid.org/0000-0002-7794-6764

\section{Ze Wang}

Dalian University of Technology

\section{Shengnan Fu}

Dalian University of Technology

\section{Chi Zhang}

Dalian University of Technology

\section{Research Article}

Keywords: Urban flood, Ponding distribution, Computer Vision, Object detection, Surveillance video

Posted Date: January 17th, 2022

DOI: https://doi.org/10.21203/rs.3.rs-1053795/v1

License: (9) This work is licensed under a Creative Commons Attribution 4.0 International License. Read Full License 


\section{Abstract}

Detection of ponding levels timely and accurately during urban floods is the basis of effective disaster prevention and mitigation. New data sources such as social media and road surveillance video record the process of urban floods in the form of images, and the development of computer vision technology bring new opportunities for extracting ponding information from these image data. This study proposes a computer vision-based method to identify the spatial-temporal distribution of ponding levels in the scene of the road surveillance video. Firstly, a dataset of sedan images compiled from three sources was collected to train an object detection algorithm, You Only Look Once vision 3 (YoloV3). Then the trained YoloV3 model was adopted to identify the ponding levels whenever and wherever sedans were detected from the videos. Secondly, the outlier detection was employed to detect and delete the outliers of ponding levels in each time step. Finally, Inverse Distance Weighted was leveraged to estimate the ponding distribution in the scene. This method was employed for two urban flood events at a street crossing, Dongguan Street, in Dalian, China. The evaluation index mAP of the trained YoloV3 model reached 78\%, which stated the model's validity. The ponding level estimated by our method was validated well with the submerged depth of a static reference, and ponding process had a strong correlation with the rainfall time series. The results can be used to analyze the process of flood rising and receding, which contributes to arrange drainage facilities and improve urban flood management.

\section{Introduction}

In the process of rapid urbanization, the underlying conditions of the city have undergone great changes, causing larger and faster hydrological responses (Wang et al. 2020). Moreover, extreme rainfall events triggered by global warming (Tabari 2020) have made several cities around globe suffer from severe urban floods frequently (Wu et al. 2020). According to the SHELDUS ( https://cemhs.asu.edu/sheldus ) database, from 1960 to 2016, direct property losses caused by floods in urban areas amounted to $\$ 107.8$ billion, affecting 20141 urban counties in the U.S. What makes this problem more complicated is that flood will move and ponding level will change over time (Alizadeh Kharazi and Behzadan 2021). Therefore, real-time dynamic ponding levels monitoring during urban floods is urgently needed, which is essential for emergency managers to prioritize relief efforts and plan effective disaster prevention and mitigation measures (Rosser et al. 2017).

Traditionally, the most common methods for monitoring urban flood information are instrumental measurement and remote sensing. The instrument measurement method is measured by various sensors with different principles, which is convenient, fast, and high precision (Huang and Shu 2016). And the disadvantage of this method is that almost no sensors are distributed on streets dedicated to urban flood monitoring (Wang et al. 2018) because of the high cost and susceptibility to external interference. As for remote sensing, it has greater space scales than other methods (Joint and Groom 2000). The obvious disadvantage is that the data obtained from remote sensing tends to have low spatial and temporal resolution (Rosser et al. 2017), which does not match the highly heterogeneous urban underlying surface 
condition (Wang et al. 2017). Recently, researchers have been finding an efficient and low-cost monitoring method to meet data accuracy requirements.

With the continuous development of information technology, the construction of "smart city" (Batty et al. 2012) has emerged. Big data is a crucial technology for supporting the realization of "smart" in all fields of smart cities (Lim et al. 2018; Bibri 2018). Attributed to the arrival of the big data era, data sources have been greatly expanded. Researchers and practitioners have begun to focus on various new data sources, such as surveillance video, social media, and applications, which create a new opportunity to collect a large amount of informative image data (Witherow et al. 2019).

Surveillance video and social media record the process of urban pluvial floods in the form of images. The application of images, usually georeferenced, time-stamped, and cost-effective, obtained by these two new data sources in monitoring urban floods has received more and more attention. For example, (Alizadeh Kharazi and Behzadan 2021) presented a deep neural network approach to estimate ponding areas from street photos. (Liu et al. 2015) manually read ponding levels from surveillance videos.

Computer vision, defined as a field of study that seeks to develop techniques to help computers "see" and understand the content of digital images such as photographs and videos, is significant technical support for realizing the application of extracting flood information from images. Computer vision is divided into five categories: image classification, object detection, target tracking, semantic segmentation, and instance segmentation (Li et al. 2020). This technology has derived many fast-growing, practical applications, such as face recognition (Sinha et al. 2006), image retrieval (Paulin et al. 2015), computer games (Hämäläinen and Höysniemi 2003), biological recognition technology (Fada et al. 2017), smart cars (Luu et al. 2019), etc.

There have been preliminary applications of computer vision in obtaining urban flood information, and they can be classified into two topics: extracting ponding areas and estimating ponding levels. For the former, (Witherow et al. 2019) proposed an image processing pipeline for detecting ponding areas on inundated roadways by registering the pair images. And (Jafari et al. 2021) leveraged object-based image analysis (OBIA) to identify ponding areas from images, and the author introduced temporal smoothness to refine the segmentation.

Considering about estimating ponding levels, two kinds of computer vision techniques have been used widely. The first kind depends on the characteristics of the image itself to identify the ponding levels using image processing algorithms. For example, (Yu and Hahn 2010) proposed a difference imagebased ponding levels measurement using sparsely sampled images in time domain, which references an indicator (an invariant feature in the image) to identify ponding levels. (Sakaino 2016) developed a twostep histogram-based method to estimate ponding levels by supervising the region of interest (ROI) from consecutive frame images. This kind of methods requires high resolution images, where obvious water surface lines can be seen, and the reliability of estimating results may be reduced in complex and noisy scenes. 
Another kind of methods is to leverage computer vision to detect reference objects in the image to identify the ponding levels indirectly. For example, (Jiang et al. 2019) employed Convolutional Neural Network (CNN) to automatically detect the reference object (traffic bucket) in flooding and non-flooding images, and the bounding box height difference of these two period images is utilized to estimate ponding levels. A similar approach was used by (Alizadeh Kharazi and Behzadan 2021), but the author developed a title correct technique to correct the error caused by the inclination of the reference objects. Some researchers tried to estimate ponding levels based on dynamic reference objects existing more widely in the scene, and they predicted a grade data of ponding levels rather than a specific value. For example, (Feng et al. 2020) leveraged Mask R-CNN combined with body keypoint detection to divide the ponding levels into five ranks. While (Chaudhary et al. 2019) improved Mask R-CNN by adding a level layer to predict ponding levels of five objects directly.

Overall, the current research primarily estimates the ponding level at a single point or the ponding area with its boundaries in the image, however, has not yet monitored the ponding distribution in the scene of the image during urban floods. It would be possible to obtain the ponding distribution by taking advantage of dynamic reference objects that can dynamically cover the whole scene. Estimating the spatial-temporal ponding distribution may have following contributions. Firstly, information assimilation can reduce the uncertainty caused by single-point information, which could improve the accuracy of predicted ponding levels in the scene. Secondly, by analyzing the difference of ponding levels at different points can give a better understanding on where the urban ponding is serious and how the flooding process is developed, which is conducive to the arrangement of drainage facilities and effective disaster prevention and mitigation.

This study aims to obtain the overall spatial-temporal urban ponding distribution from surveillance videos with the help of computer vision technique. The proposed method makes full use of the dynamic movement characteristic of the dynamic reference object. The remainder of this paper is organized as follows: Section 2 introduces the study area and data collected; Section 3 describes the method proposed, including ponding levels identification, outlier detection, and ponding distribution determination; Section 4 shows the evaluation results of trained object detection model and the estimated ponding distribution map in two cases; Section 5 mainly discusses the findings and limitations; and the conclusion is given in Section 6.

\section{Study Area And
2.1 Study area}

In order to study the spatial-temporal distribution of ponding levels in urban street scenes, we selected Dongguan Street in Dalian, China, as the experimental site (Fig. 1). The central city of Dalian, located at the southernmost tip of the Liaodong Peninsula in China, is an essential coastal port city in Liaoning Province, China. Due to the northward typhoon or strong convective weather, Dalian is prone to flood 
disasters from July to August. And Dongguan Street is one of the waterlogging prone points mentioned in the Dalian Planning Report.

\section{Place Fig. 1 here}

\subsection{Data}

A video data set from the traffic management department on Dongguan Street was collected to estimate the image-based ponding levels during urban floods. The videos recorded two urban flood events on August 13, 2019, and August 13, 2020. The details of these two videos are shown in Table 1. The video data set was intercepted every five seconds into 1057 images in the Joint Photographic Experts Group (JPEG) format. The original resolution of these images is $1200 \times 720$ pixels.

Table 1

Two videos in the surveillance video data set

\begin{tabular}{|lllll|}
\hline & Data & Timeframe & Number of images & Resolution \\
\hline Video 1 & August 13, 2019 & $16: 40-17: 40$ & 533 & $1200 \times 720$ \\
\hline Video 2 & August 13, 2020 & $15: 30-16: 15$ & 524 & $1200 \times 720$ \\
\hline
\end{tabular}

In order to evaluate the rationality of the spatial-temporal ponding distribution, this study collected the rainfall data of two periods corresponding to the videos. In addition, the ponding levels estimated by the static reference, the guardrail, was used for verification. By visually observing the Region of interest (ROI) images where the static reference object is located, we estimated the pixel height of the selected static reference and calculated the actual height of each pixel according to the actual height of the guardrail. The difference between guardrail heights in paired flooding and non-flooding images was regarded as the water depth. Fig. 2 presents the time series of these two pluvial flood events and the water depth time series estimated by the static reference.

Place Fig. 2 here

\section{Methodology}

In this study, a computer vision-based method is proposed to identify the spatial-temporal distribution of ponding levels in the scene of the road surveillance video, which is shown in Fig. 3. The three main tasks of this method are as follows:

i) Ponding levels This task used images of obtained sedans to train an object detection model, You Only Look Once vision 3 (YoloV3), and employed the trained model to predict ponding levels and locations whenever and wherever sedans were detected from consecutive video images.

ii) Outlier In order to reduce the uncertainty of single-point detection, the outlier detection technology was implemented to analyze the outliers of ponding levels value in each time step, and the outliers were deleted to update the points with locations and ponding levels. 
iii) Ponding distribution In this task, Inverse Distance Weighted was leveraged to estimate the ponding distribution in the scene.

Place Fig. 3 here

\subsection{Ponding levels identification}

Identifying ponding levels based on dynamic reference objects from consecutive surveillance video images is an important part of this study. Since this task aims to quantify the ponding levels based on reference objects partially submerged in the water, the first step is to decide which objects we should consider for the classification task. Due to the limited scalability of static reference objects, we selected the sedan as the dynamic reference for the ponding levels identification in this study. According to the dynamic reference submerged degree during floods in the images, the ponding levels was labeled in four classes. Then, the labeled images were used to train the object detection model, YoloV3, to identify the ponding levels of the test images, and a mean average precision (mAP) was used to evaluate the model performance. After that, sedans with the locations and ponding levels in consecutive images from surveillance videos were detected by the trained YoloV3 model.

\subsubsection{Training dataset and annotation strategy}

The training dataset consisting of three sources images was collected to train YoloV3 model, shown in Table 2. In order to ensure the size of the dynamic reference object is basically the same, the sedan (not the bus and SUV car) was selected in our study. We chose images containing sedans as our training dataset, which are collected from three sources: media images, synthetic images, and surveillance video images. We searched Google with 'flood sedan' as the keyword and got 292 pictures. For synthetic images, we truncated the reference object image with different heights to represent that part of them is visible on the water surface during flood (Jiang et al. 2020). We created 11 groups of different angles sedan images, and the sedans of each group had 13 different truncation heights. Finally, we created 143 images by merging the sedans on a water surface image in a blending way. The 405 surveillance video images were collected from the Dongguan Street video screenshots obtained through the traffic management department. 
Table 2

Training dataset

\begin{tabular}{|lllllll|}
\hline \multirow{2}{*}{ Type } & Number of images & \multicolumn{4}{l|}{ Number of labels per level } \\
\cline { 3 - 7 } & & Level 0 & Level 1 & Level 2 & Level 3 & total \\
\hline Media images & 292 & 100 & 69 & 112 & 93 & 374 \\
\hline Synthetic images & 143 & 11 & 22 & 44 & 66 & 143 \\
\hline Surveillance video & 405 & 29 & 436 & 140 & 105 & 710 \\
\hline Total & 840 & 140 & 527 & 296 & 264 & 1227 \\
\hline
\end{tabular}

In this paper, sedans in our image dataset were labeled with ponding levels and bounding boxes to train the object detection. Since humans cannot infer the centimeters of the flood just by observing the images, we tried to estimate the extent of sedans submerged in the water according to roughly defined ponding levels (Chaudhary et al. 2019). Considering the proportion of cars in surveillance video and the impact of ponding levels on the traffic, we defined 4 ponding levels, which range from 0 to 3 . Level 0 means no water; Level 1 means ponding levels below 1/3 of the wheels; Level 2 means ponding levels below the fog light of sedans; Level 3 means ponding levels above the fog light, which will cause the vehicle in danger during floods. The labellmg (https://github.com/tzutalin/labellmg) software was used to label the collected images, and each image had two attributes: the label of ponding levels and the bounding box. Fig. 4 shows each level at the corresponding height on the sedan and representative pictures from three data sources.

\section{Place Fig. 4 here}

\subsubsection{The object detection model -YoloV3}

YoloV3 was used as the object detection model in our study, which is based on Convolutional Neural Network (CNN) and has the advantage of being much faster than other networks and still maintains accuracy (Martinez-Alpiste et al. 2021). The backbone network of YoloV3 is Darknet-53, which is the feature extractor. The rest detection layer, named YOLO layer, is to predict objects at three scales. The detail of YoloV3 can be seen in (Redmon and Farhadi 2018). The official pre-trained model weights based on the MS COCO (Microsoft Common Objects in Context) dataset (Lin et al. 2014) can be used before training the model. The COCO dataset is large-scale object detection, key-point detection, segmentation, and captioning dataset consisting of $328 \mathrm{~K}$ images. For object detection, the COCO dataset has annotations for 80 object categories.

In our task, the training was divided into two stages: in the first stage, we loaded the official pre-trained weights as the initial weights. Moreover, due to the backbone feature extraction layer is suitable for our data set, we chose to freeze the backbone network and just trained the rest detection layer during the first 50 epochs to protect them from large weight perturbations during the early phases of the new training, thereby achieving higher accuracy in a shorter period of training. In the second stage, we leveraged the 
weights that had been trained in the first stage to continue training. Different from the first stage, we unfroze all layers and trained all weights. The learning rates of the two stages were 0.1 and 0.02 , respectively, and batch-size were 8 and 4 . Of the 840 images we got, $80 \%$ were used as the training set for model training mentioned above, and $20 \%$ were used as the test set for verification. We stopped training in the 2000 epoch when the final loss value no longer decreased.

\subsubsection{Evaluation strategy}

In this task, the mean average precision (mAP) was used to evaluate our object detection model (Gaus et al. 2019). In multiple categories of object detection, by adjusting the Intersection Over Union (IOU) threshold, we can draw the P-R curve for each object of our model, and the area of the P-R curve is the average precision (AP).

\subsubsection{Ponding levels detection}

Using the trained YoloV3 model, the ponding levels and the bounding box can be obtained wherever the sedan is detected in the consecutive surveillance video images. In addition, it should be mentioned that the midpoints of the bottom border of the detected bounding boxes can represent the location of the identified ponding points to ensure that they can fall on the road.

\subsection{Outlier detection}

Outlier detection in our method was used to detect and delete the outlier points in each time step. For our data, we defined the time step as five minutes and proposed a hypothesis that the state of ponding levels every five minutes is basically unchanged. After predicting the ponding levels at special points, we put points in every five minutes together. However, because of the limited accuracy of object detection, some of the ponding levels in every five minutes may be misidentified. Therefore, data mining needed to be adopted to reduce uncertainty. This paper leveraged the outlier detection method to realize data mining.

To eliminate these spatial outliers, we decided to use Cluster and Outlier Analysis (Anselin Local Moran's I). For outliers, different elements will be adjacent or surrounded by other elements in geographic space, so the following description is used: the high value surrounded by low values is expressed as $\mathrm{HL}$; on the contrary, a low value surrounded by high values is expressed as LH. The local Moran index (local Moran's I) indicates the distribution of spatial values (Anselin 1995). A negative local Moran's I indicates that the element is an outlier. Local Moran's I is defined as following:

$$
I_{i}=\frac{x_{i}-\bar{X}}{S_{i}^{2}} \sum_{j=1, j \neq i}^{n} w_{i, j}\left(x_{j}-\bar{X}\right)
$$

$$
S_{i}^{2}=\frac{\sum_{j=1, j \neq i}^{n}\left(x_{j}-\bar{X}\right)}{n-1}
$$


Where $x_{i}$ presents the attribute value of element i, $X$ presents the average value of the corresponding attribute, $w_{i, j}$ presents Spatial weight between elements $\mathrm{i}$ and $\mathrm{j}, \mathrm{n}$ presents the total number of elements.

To apply this method in ArcGIS, we mainly choose two parameters. The Fixed-Distance-Band is used as the representation of the spatial relationship of feature points, which means neighboring features within the specified critical distance should be assigned the same weight, and the features above threshold distance cannot affect the detection feature. In addition, we chose Euclidean-Distance to calculate the distance between each element and its neighboring elements.

\subsection{Ponding distribution determination}

After detecting and deleting outliers in each time step, the update points can basically represent ponding levels at specific locations. Then, to acquire the ponding distribution in the entire scene, Inverse Distance Weighted (IDW) was used for spatial interpolation. Finally, water depth time series based on static reference detection supported verifying the rationality of spatial-temporal ponding inundation maps.

\subsubsection{Inverse Distance Weighted (IDW)}

Inverse distance weight (IDW) interpolation was implemented to estimate the ponding distribution maps based on the update points in every time step in our study. IDW uses a linear weight combination of sampling points to determine the cell value (Watson and Philip 1985). The weight is an inverse distance function. This method assumes that the mapped variable is reduced due to the distance between its sampled location. This interpolation method is suitable for the scattered nodes, which is consistent with our data.

We used ArcGIS to perform inverse distance weighted interpolation, which has two key parameters. One is the radius value, which is used to characterize the range of data points included in the interpolation calculation. We chose Variable Search Radius as the radius value. The points number in calculating the interpolated cell value was specified as 18 according to our data size. Another parameter is the Power, which is used to characterize the weight difference of data points at different distances from the sampling position in the interpolation calculation. We chose 3 as the Power parameter in our task.

\subsubsection{Verification of the Spatial-temporal ponding distribution}

In order to verify the rationality of spatial-temporal ponding distribution, this paper selected rainfall data and water depth time series identified based on static reference for comparative analysis. The detail of two validation data is mentioned in Section 2.

\section{Results}




\subsection{Ponding levels identification}

A mean average precision (mAP) index was used to evaluate the trained model performance on the test set, 168 images. The P-R curves of different levels are shown in Fig. 5(a), and mAP can be seen in Fig. 5 (b). The AP of level 3 was as high as $91 \%$, and the AP of level 2 is up to $82 \%$, which indicates that this task performs well at level 2 and 3 , that is, the high level. While the APs of level 1 and level 0 were $64 \%$ and $74 \%$, respectively, which were lower than mAP. Fig. 5(c) shows the proportions of False Positive (FP) and True Positive (TP) of the detection results per level when the score threshold is 0.5 . It can be seen that TPs of level 1, 2 and 3 had high proportion, indicating that the detection of these three levels had high precision. Combined with the annotation strategy, it could conclude that the reason why level 0 performance poorly is that the dividing line between level 0 and level 1 is not apparent, especially when there is no water on the ground but not completely dry. Overall, the mAP had reached $78 \%$ in the test set, and it stated the model's validity.

\section{Place Fig. 5 here}

The accuracy of the trained YoloV3 model was $78 \%$, not $100 \%$, which indicated the uncertainty of singlepoint object detection. It also pointed out the necessity of performing outlier detection before ponding distribution determination.

\subsection{Outlier detection}

Outlier detection was leveraged to detect the outliers of all points every five minutes to verify the uncertainty of the single-point object detection. We gathered the detected points with ponding levels and locations every five minutes, as shown in Fig. 6 . The detected HL points were the level 1 points, marked in red, surrounded by level 0 points, which can be considered as outliers. Similarly, some low values marked in blue surrounded by high values were detected as LH values, which were also the points of false detection.

\section{Place Fig. 6 here}

We counted the number of objects identified in every five minutes of the two cases and the number of outliers to prove the rationality of deleting outliers. As shown in Fig. 7, we can conclude that outliers had a low proportion, although they existed. The number of objects every five minutes was different, ranging from 26 to 144, which can be interpreted as the difference of traffic flow in different times in a real urban road scene. The total HL and LH points every five minutes are shown in Fig. 7 (right), where blue represents LH points and orange represents HL points. The proportion of outliers in each time step was almost below $20 \%$. According to the statistical data, it can be concluded that single point detection is uncertain. After deleting all outliers, we obtained updated points with higher accuracy.

\section{Place Fig. 7 here}

\subsection{Ponding distribution determination}


We got the five-minute ponding distribution maps of the two cases through data interpolation, shown in Fig. 8 and Fig. 9. In case 1, the degree of flooding gradually increased before 17:00, and the ponding levels reached level 3 from 17:00 to 17:25. After 17:25, the degree of inundation gradually decreased. The bridge tunnel on both sides reached level 3 at 17:15, which was the most severe moment during this urban flood event. In case 2, the flood inundation degree gradually increased before $15: 45$, and the ponding levels of the right bridge tunnel continuously reached level 3 from 15:45 to 15:55. The flooding degree gradually decreased after 15:55. In the whole process, the left bridge tunnel did not reach level 3 . At the same time, we found that the right bridge tunnel reached its peak earlier than the left in both cases.

The ponding process estimated by our method strongly correlated with the rainfall time series, shown in Fig. 8 and Fig. 9. Fig. 8 expresses the spatial ponding distribution in Case 1. The top of each group in graphs is the corresponding rainfall during this time step, marked in blue. It can be seen that some areas in the scene reached level 3 from 17:00 to 17:20. According to the rainfall time series shown in Fig. 8, we can see that the rainfall is relatively large from 16:50 to 17:15, with two rain peaks. In addition, due to the time lag between rainfall and the formation of urban floods, it can be concluded that the flooding process was basically the same as the rainfall time series. Fig. 9 reflects the spatial ponding distribution in Case 2. It can be seen that only in two time steps of $15: 45$ and 15:50, the ponding levels in certain areas reached level 3 , and they appeared after peak rainfall, which was corporate with the pattern of urban flood formation.

By observing the evolution of flood in Case 2, it can be seen that ponding level under the bridge tunnel suddenly dropped from level 3 to level 1 at 15:55, and the ponding levels returned to level 2 at 16:40. This phenomenon was inconsistent with the continuity of the ponding process. We found that the camera lens had water droplets, which affected the detection of the upper left area of the scene.

\section{Place Fig. 8 here}

\section{Place Fig. 9 here}

Through the ponding distribution maps, we can find the evolution pattern of urban floods in this scene. Comparing with the distribution maps in Fig. 8 and Fig. 9, we can see that the ponding levels in the left bridge tunnel always reached the highest level first than that in the right bridge tunnel, which may be interpreted as the decline of the drainage pipe network capacity at the right bridge tunnel. This information also provides a reference for future disaster prevention and mitigation measures. For example, the situation under the right bridge tunnel should be focused. At the same time, we can see that the ponding levels in the lower area of the images were always level 1 or below, which is consistent with the topography of this scene that the terrain under the bridge hole is lower than the surrounding area.

In order to further verified the rationality of our results, we compared ponding levels based on ponding distribution maps with the detected water depth time series based on the static reference object (mentioned in Section 2), as shown in Fig. 10. We extracted the ponding levels at the location of the static reference object in the ponding distribution map and converted the ponding levels to the corresponding 
water depth, shown by the blue step line. It can be concluded that the trend of the ponding level process was basically the same in two situations, with the maximum ponding levels appearing at 17:10-17:15 in Case 1 and 15:50-15:50 in Case 2. It is acceptable that the ponding levels curves obtained by the two methods cannot wholly coincide due to the influence of interpolation and microtopography.

\section{Place Fig. 10 here}

\section{Discussion}

Through our research, it can be concluded that the accuracy of object detection is the core reason that affects the results. In our task, the accuracy of the trained model was $78 \%$. This paper compares the performance of the four widely used object detection models (Faster-RCNN, R-FCN, SSD, YoloV3). According to the test results on Microsoft COCO object detection dataset presented in (Liu et al. 2016; Dai et al. 2016; Ren et al. 2017; Redmon and Farhadi 2018), the mAP @ [.5:.95] of Faster-RCNN, R-FCN, SSD (512*512 input image), YoloV3 (608*608 input image) are $21.9 \%, 31.5 \%, 26.8 \%, 33.0 \%$. Because of the complexity of the task, the object categories, and different settings, the mAPs of these four models are lower than that of our model. Therefore, it is difficult to make a fair comparison between different object detection models, and we should treat the evaluation results rationally (Huang et al. 2016). The main factors affecting accuracy can be divided into the following aspects: 1) feature extractors; 2) input image resolutions; 3) data augmentation; 4) training dataset; 5) training configurations including batch size, learning rate, learning rate decay, etc.; and 6) environmental factors. For video surveillance images, environmental conditions are also important factors affecting model performance, such as strong shadows, bright lights, obstructions, night scenes, and dirt or dust on the camera lens.

In our context, the main influence factors are environmental factors. In order to better explain the reasons that arise the wrong classification in our experiments, some representative wrongly classified images are shown in Fig. 11. The main reasons can be divided into four categories: 1) high beams influence: when the high beam is turned on at night, the target object could become challenging to distinguish affected by the light (Fig. 11(b), not detected); 2) obstacles in front of the predicted object: there will inevitably be overlapping vehicles (the bus and the car in Fig. 11(a)) under the lens. Therefore, when a part of the target object is blocked by other vehicles or objects, the classification will be interfered; 3 ) influence of vehicle angle: when the vehicle is in a frontal view, that is, when the front or rear of the vehicle is facing the camera (Fig. 11(c)), the characteristics such as wheels become inconspicuous, resulting in the wrong classification; and 4) impact of water splash: when the vehicle speed is too fast to cause water splash, a vehicle that should be in level 1 is easily misjudged as level 2 (Fig. 11(d)).

\section{Place Fig. 11 here}

There are still some limitations in this study, and they are expected to be solved in the future. First, due to the limitation of the data obtained, the experiments in this paper are limited to a single scene. In the future study, the accuracy of this model in multiple scenes will verify the transferability of our method. In addition, we proposed a hypothesis that the state of ponding levels every five minutes is basically 
unchanged. How to fully mine spatial-temporal comprehensive information to obtain a more refined ponding distribution map will be a direction of future work. Finally, this paper only applies to video surveillance images. In the future, social media images and other crowdsourced data with geographic labels and time can be combined to obtain a larger scale of ponding distribution, which is also an effective way to realize the smart city.

\section{Conclusion}

It is very significant to obtain timely urban flood information for disaster prevention and mitigation. In this study, a computer vision-based approach to estimate the spatial-temporal ponding distribution from road surveillance video images was proposed and verified, which takes advantage of the dynamic movement characteristics of the dynamic reference object. An object detection model called YoloV3 was utilized to predict ponding levels whenever and wherever sedans were detected from the videos. An outlier detection model was proposed to analyze and delete the outliers in each time step to limit the error of the single point result of the object detection. Furthermore, Inverse distance weight (IDW) interpolation was leveraged to infer the ponding distribution of the entire area from multiple single-point ponding levels.

The experiments have proved the ability of object detection model YoloV3 to identify ponding levels from images, and the evaluation index mAP reached $78 \%$. Moreover, the proportion of outliers was almost below $20 \%$, verifying the necessity and rationality of outlier detection. And the ponding process estimated by our method had a strong correlation with the rainfall time series, with a five-minute time lag. After comparing ponding levels based on ponding distribution maps and the detected water depth time series based on the static reference object, the trend of these two curves was basically the same, with the maximum ponding levels appearing at the same time step. Meanwhile, through the ponding distribution maps, we can find the evolution pattern of flood in this scene, which contributes to the arrangement of drainage facilities and effective disaster prevention and mitigation.

\section{Declarations}

\section{Authors Contributions}

For this research paper with several authors, a short paragraph specifying their individual contributions was provided. Xin Hao collected the data, performed the calculations, and wrote the first manuscript. Heng Lyu conceived the original idea, helped supervise the study and revised the manuscript. Ze Wang helped carry out the calculations. Shengnan Fu helped collect the data. Chi Zhang supervised the project. All authors discussed the results and contributed to the final manuscript.

\section{Availability of data and material}

The models and code that support the findings of this study are available from the corresponding author upon reasonable request. 


\section{Ethical Approval}

This article does not contain any studies with human participants or animals performed by any of the authors.

\section{Conflict of interest}

The authors declare that there is no conflict of interest.

\section{References}

1. Alizadeh Kharazi B, Behzadan AH (2021) Flood depth mapping in street photos with image processing and deep neural networks. Comput Environ Urban Syst 88:101628. doi: 10.1016/j.compenvurbsys.2021.101628

2. Anselin L (1995) Local Indicators of Spatial Association-LISA. GEOGR ANAL 27(2):93-115

3. Batty M, Axhausen KW, Giannotti F, Pozdnoukhov A, Bazzani A, Wachowicz M, Ouzounis G, Portugali Y (2012) Smart cities of the future. The European Physical Journal Special Topics 214(1):481-518. doi: 10.1140/epjst/e2012-01703-3

4. Bibri SE (2018) The loT for smart sustainable cities of the future: An analytical framework for sensorbased big data applications for environmental sustainability. SUSTAIN CITIES SOC 38:230-253. doi: 10.1016/j.scs.2017.12.034

5. Chaudhary P, Aronco D, Moy De Vitry S, Leitão M, Wegner JP JD (2019) FLOOD-PONDING LEVELS ESTIMATION FROM SOCIAL MEDIA IMAGES. ISPRS annals of the photogrammetry, remote sensing and spatial information sciences, IV-2/W5, 5-12. 10.5194/isprs-annals-IV-2-W5-5-2019

6. Dai J, Li Y, He K, Sun J (2016) R-FCN:Object Detection via Region-based Fully Convolutional Networks

7. Fada JS, Hossain MA, Braid JL, Yang S, Peshek TJ, French RH (2017-01-01) Electroluminescent Image Processing and Cell Degradation Type Classification via Computer Vision and Statistical Learning Methodologies, 2017. IEEE, p 3456-3461. doi: 10.1109/PVSC.2017.8366291

8. Feng Y, Brenner C, Sester M (2020) Flood severity mapping from Volunteered Geographic Information by interpreting ponding levels from images containing people: A case study of Hurricane Harvey. ISPRS J PHOTOGRAMM 169:301-319. doi: 10.1016/j.isprsjprs.2020.09.011

9. Gaus YFA, Bhowmik N, Akcay S (2019) Evaluating the Transferability and Adversarial Discrimination of Convolutional Neural Networks for Threat Object Detection and Classification within X-Ray Security Imagery. IEEE. doi: 10.1109/ICMLA.2019.00079

10. Hämäläinen P, Höysniemi J (2003) A Computer Vision and Hearing Based User Interface for a Computer Game for Children (299-318). Berlin, Heidelberg: Springer Berlin Heidelberg. (Reprinted. doi: 10.1007/3-540-36572-9_24 
11. Huang B, Shu X (2016) Ultra-compact strain- and temperature-insensitive torsion sensor based on a line-by-line inscribed phase-shifted FBG. OPT EXPRESS 24(16):17670. doi: 10.1364/OE.24.017670

12. Huang J, Rathod V, Sun C, Zhu M, Korattikara A, Fathi A, Fischer I, Wojna Z, Song Y, Guadarrama S, Murphy K (2016) Speed/accuracy trade-offs for modern convolutional object detectors

13. Jiang J, Liu J, Cheng C, Huang J, Xue A (2019) Automatic Estimation of Urban Waterlogging Depths from Video Images Based on Ubiquitous Reference Objects. REMOTE SENS-BASEL 11(5):587. doi: $10.3390 /$ rs 11050587

14. Jiang J, Qin C, Yu J, Cheng C, Liu J, Huang J (2020) REMOTE SENS-BASEL 12(6):1014. doi: 10.3390/rs12061014. Obtaining Urban Waterlogging Depths from Video Images Using Synthetic Image Data

15. Joint II, Groom SB (2000) Estimation of phytoplankton production from space: current status and future potential of satellite remote sensing. J EXP MAR BIOL ECOL 250(1-2):233-255. doi: 10.1016/s0022-0981(00)00199-4

16. Li T, Jiang C, Bian Z, Wang M, Niu X (2020) Semantic Segmentation of Urban Street Scene Based on Convolutional Neural Network. Journal of physics. Conference series, 1682(1). doi: 10.1088/1742$6596 / 1682 / 1 / 012077$

17. Lim C, Kim K, Maglio PP (2018) Smart cities with big data: Reference models, challenges, and considerations. Cities 82:86-99. doi: 10.1016/j.cities.2018.04.011

18. Lin TY, Maire M, Belongie S, Hays J, Perona P (2014) Microsoft COCO:Common Objects in Context. ECCV

19. Liu L, Liu Y, Wang X, Yu D, Liu K, Huang H, Hu G (2015) Developing an effective 2-D urban flood inundation model for city emergency management based on cellular automata. NAT HAZARD EARTH SYS 15(3):381-391. doi: 10.5194/nhess-15-381-2015

20. Liu W, Anguelov D, Erhan D (2016) SSD: Single Shot MultiBox Detector. European Conference on Computer

21. Luu DL, Lupu C, Chirita D (2019-01-01) Design and Development of Smart Cars Model for Autonomous Vehicles in a Platooning, 2019. IEEE, p 21-24. doi: 10.1109/EMES.2019.8795199

22. Martinez-Alpiste I, Golcarenarenji G, Wang Q, Alcaraz-Calero JM (2021) A dynamic discarding technique to increase speed and preserve accuracy for YOLOv3. 10.1007/s00521-021-05764-7

23. Paulin M, Douze M, Harchaoui Z, Mairal J, Perronin F, Schmid C (2015-01-01) Local Convolutional Features with Unsupervised Training for Image Retrieval, 2015. IEEE, p 91-99. doi:

10.1109/ICCV.2015.19

24. Redmon J, Farhadi A (2018) YOLOv3:An Incremental Improvement

25. Ren S, He K, Girshick R, Sun J (2017) Faster R-CNN: Towards Real-Time Object Detection with Region Proposal Networks. IEEE Trans Pattern Anal Mach Intell 39(6):1137-1149. doi:

10.1109/TPAMI.2016.2577031 
26. Rosser JF, Leibovici DG, Jackson MJ (2017) Rapid flood inundation mapping using social media, remote sensing and topographic data. NAT HAZARDS 87(1):103-120. doi: 10.1007/s11069-0172755-0

27. Sakaino H (2016) Camera-Vision-Based Ponding levels Estimation. IEEE SENS J 16(21):7564-7565. doi: $10.1109 /$ JSEN.2016.2603524

28. Sinha P, Balas B, Ostrovsky Y, Russell R (2006) Face Recognition by Humans: Nineteen Results All Computer Vision Researchers Should Know About. P IEEE, 94(11), 1948-1962. doi:

10.1109/JPROC.2006.884093

29. Tabari $\mathrm{H}$ (2020) Climate change impact on flood and extreme precipitation increases with water availability. SCI REP-UK 10(1). doi: 10.1038/s41598-020-70816-2

30. Wang J, Hu C, Ma B, Mu X (2020) Rapid Urbanization Impact on the Hydrological Processes in Zhengzhou. China WATER-SUI 12(7):1870. doi: 10.3390/w12071870

31. Wang R, Mao H, Wang Y, Rae C, Shaw W (2018) Hyper-resolution monitoring of urban flooding with social media and crowdsourcing data. COMPUT GEOSCI-UK 111(C):139-147. doi: 10.1016/j.cageo.2017.11.008

32. Wang Y, Di Sabatino S, Martilli A, Li Y, Wong MS, Gutiérrez E, Chan PW (2017) Impact of land surface heterogeneity on urban heat island circulation and sea-land breeze circulation in Hong Kong. Journal of Geophysical Research: Atmospheres 122(8):4332-4352. doi: 10.1002/2017JD026702

33. Watson DF, Philip GM (1985) A refinement of inverse distance weighted interpolation. GeoProcessing

34. Witherow MA, Sazara C, Winter-Arboleda IM, Elbakary MI, Cetin M, Iftekharuddin KM (2019) Floodwater detection on roadways from crowdsourced images. Computer Methods in Biomechanics and Biomedical Engineering: Imaging \& Visualization 7(5-6):529-540. doi:

10.1080/21681163.2018.1488223

35. Wu Z, Zhou Y, Wang H, Jiang Z (2020) Depth prediction of urban flood under different rainfall return periods based on deep learning and data warehouse. SCI TOTAL ENVIRON 716:137077. doi: 10.1016/j.scitotenv.2020.137077

36. Yu J, Hahn H (2010) Remote Detection and Monitoring of a Ponding levels Using Narrow Band Channel. J INF SCI ENG 26(1). doi: 10.6688/JISE.2010.26.1.6

\section{Figures}

\section{Figure 1}

Study area 
Case 1

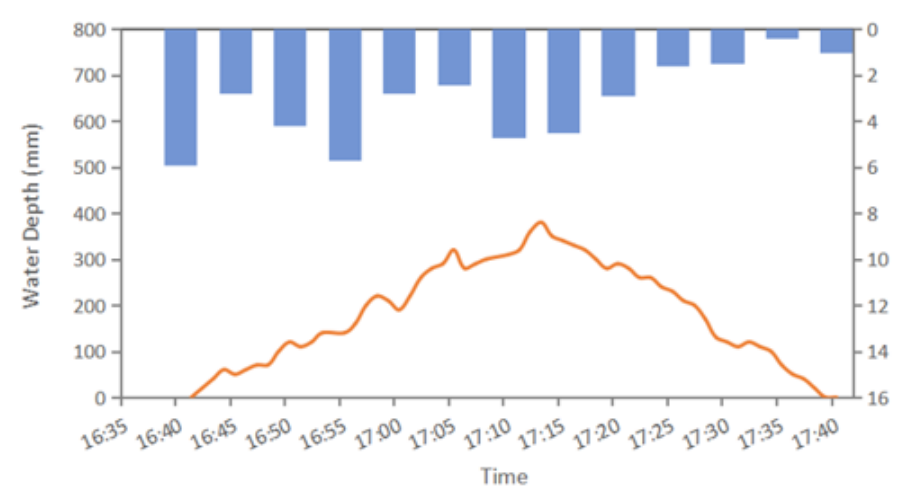

Case 2

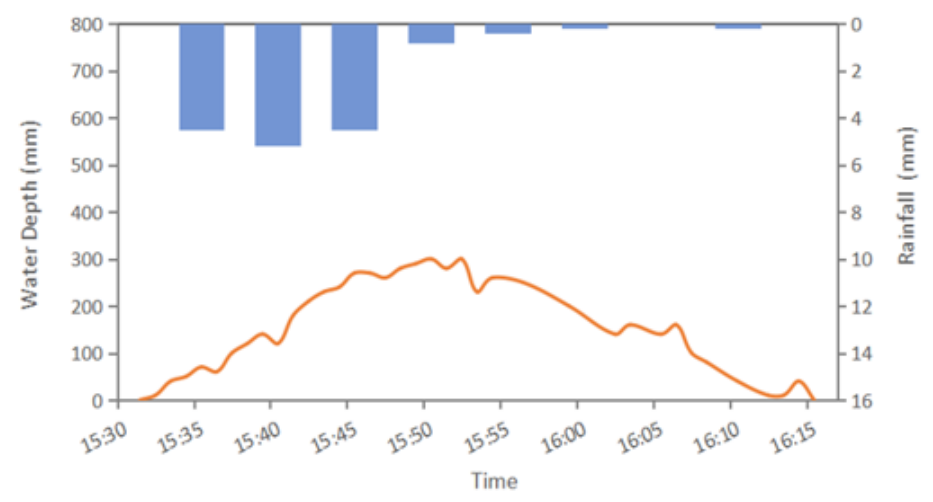

Figure 2

Rainfall time series and water depth estimated by the static reference of two pluvial flood events on August 13, 2019 and August 13, 2020, in Dalian.

\section{Figure 3}

Framework of the proposed method for computer vision-based spatial-temporal distribution of ponding levels estimation.

\section{Figure 4}

The annotation strategy for sedans (up) and representative pictures from three data sources 

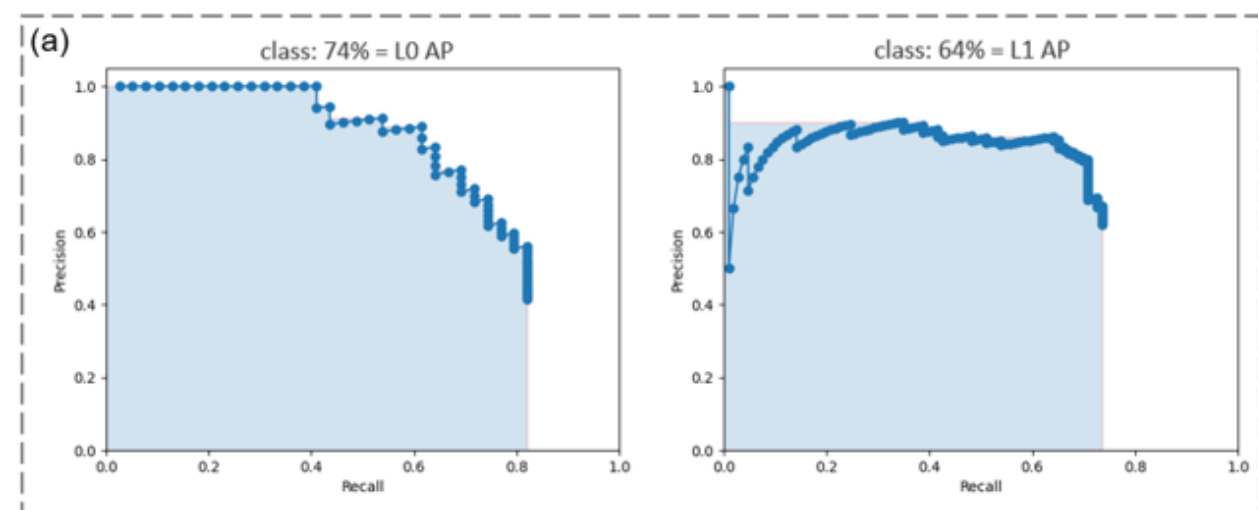

(b)

$\mathrm{mAP}=78 \%$

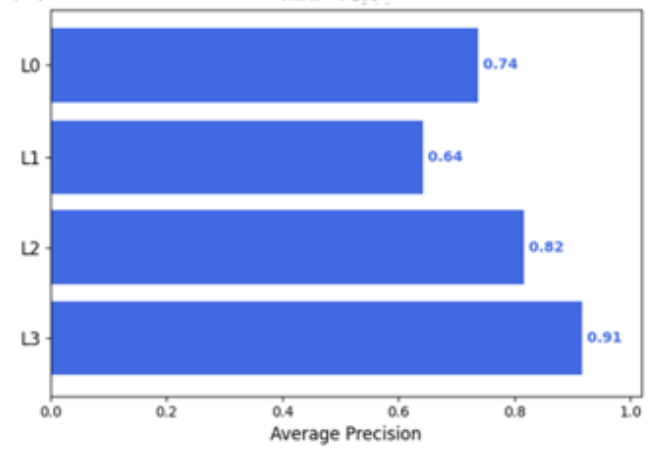

(c)
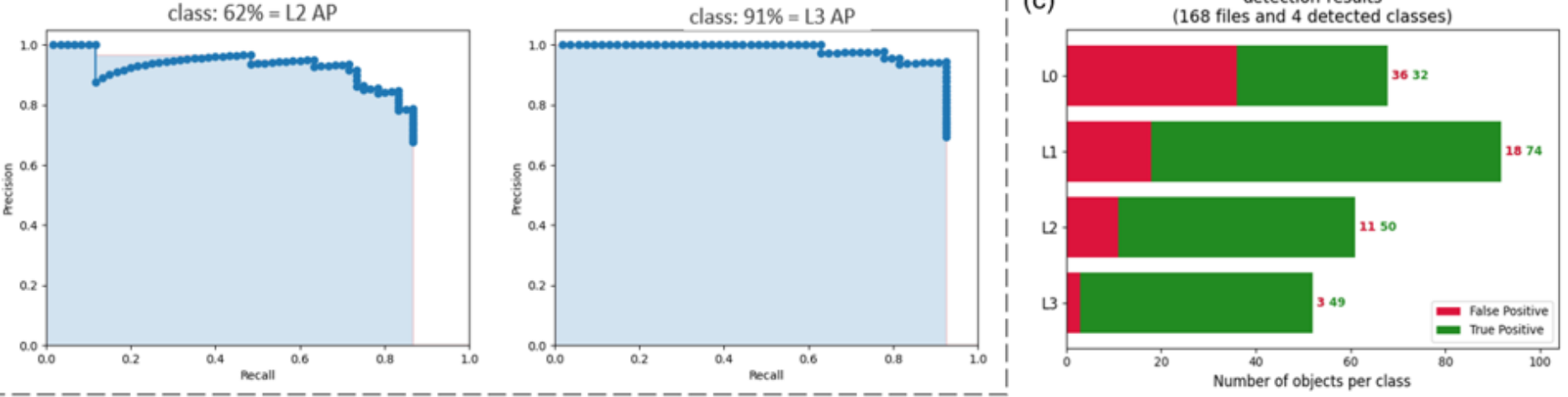

Figure 5

The evaluation results of the trained model: (a) the P-R curves of each level; (b) AP of per class: Level 3 is the highest, $91 \%$, Level 1 is the lowest, $64 \%$ and $\mathrm{mAP}$ is $78 \%$; (c) the False Positive and True Positive of the detection results per class on the test set. 


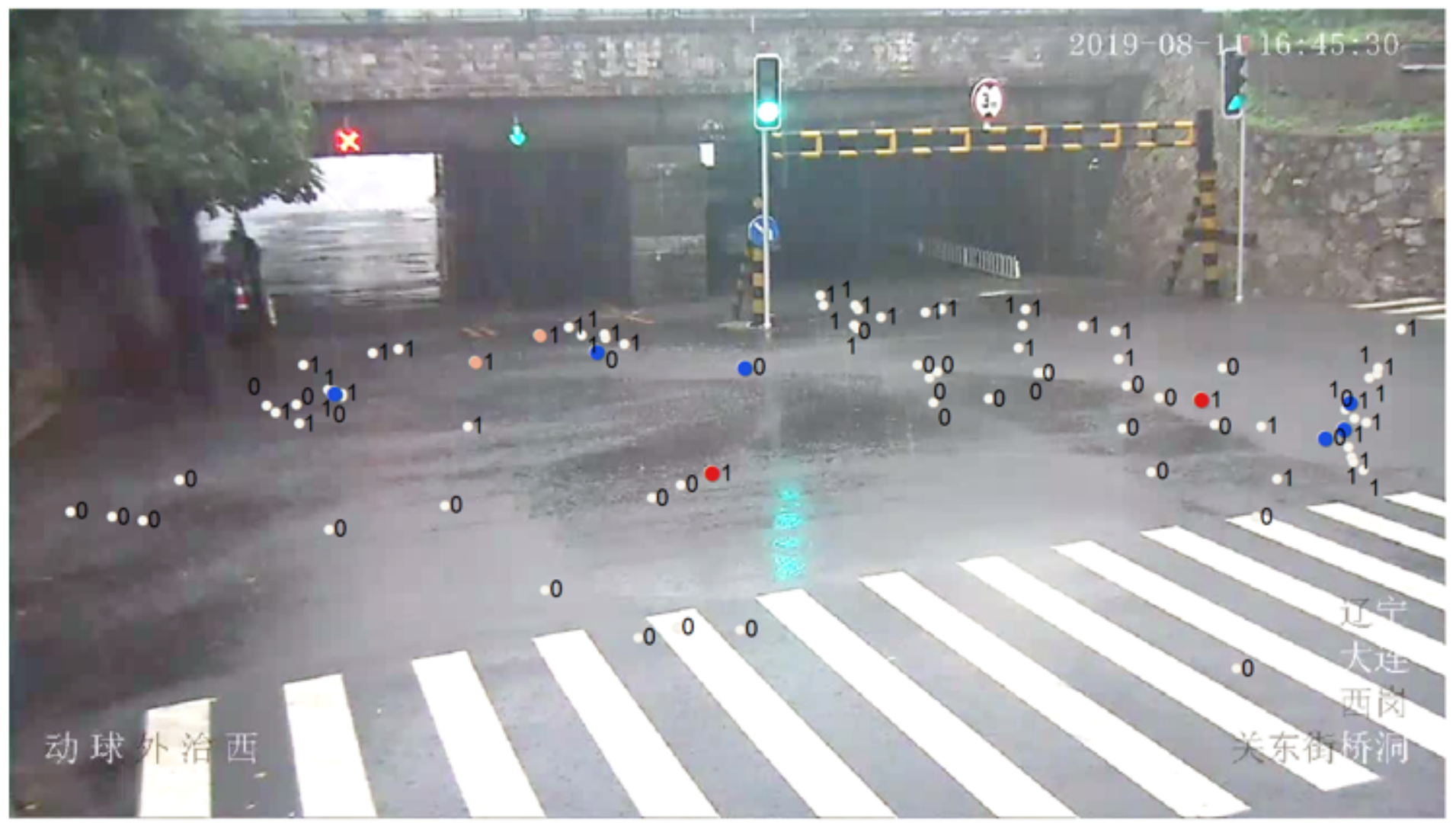

\section{Figure 6}

The outlier detection results in one time step. The white points indicate the locations and the corresponding detected ponding level of the identification points by the trained YoloV3 model. The detected HL points are marked with red, and the detected LH points are marked with blue. 
The data number of Case 1

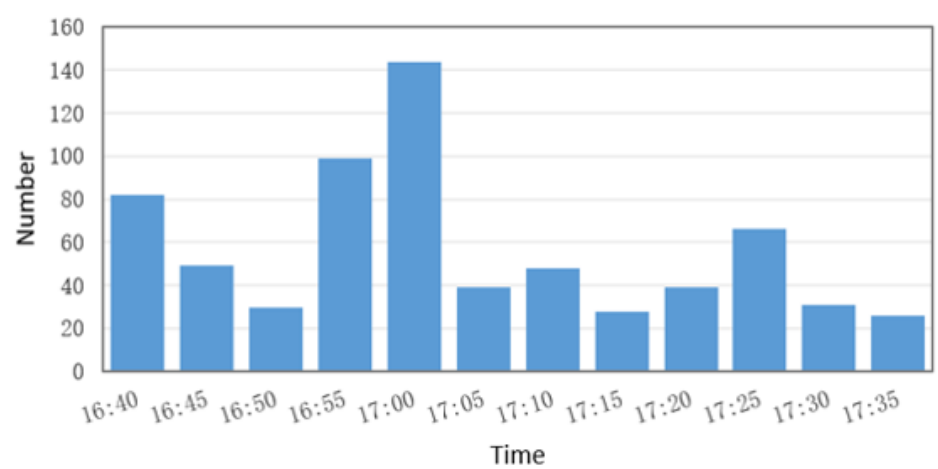

The data number of Case 2

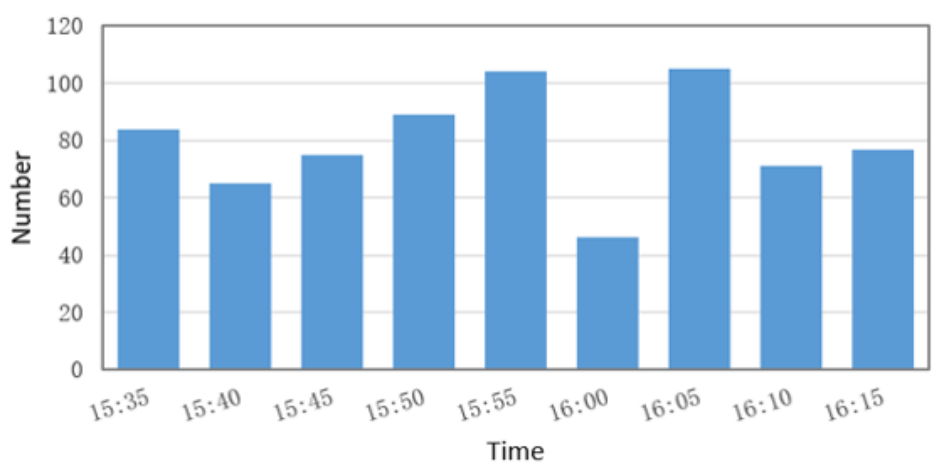

Proportion of Outlier points (Case 1)
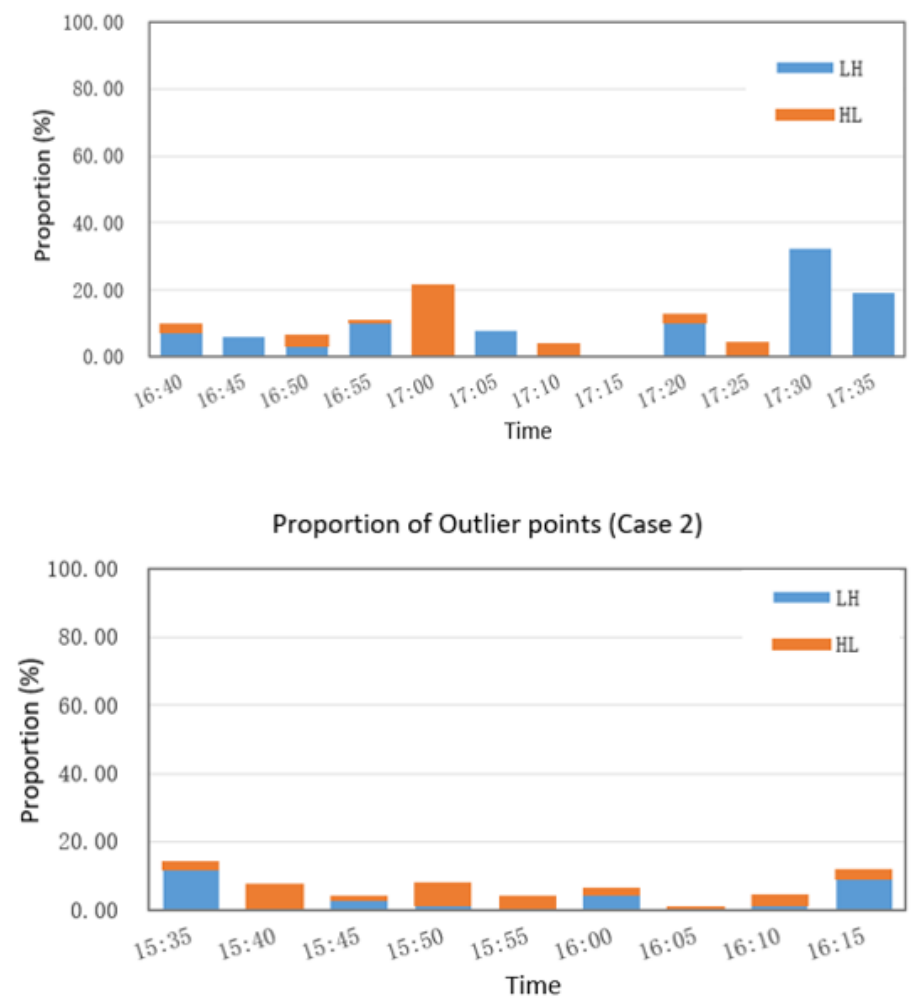

\section{Figure 7}

The number of objects identified in each time step of two cases (left) and the proportion of outlier points (right): the orange histograms represent $\mathrm{HL}$ points proportion per time step, the blue histograms represent LH points proportion per time step, and the proportion of outliers in each time step is almost below $20 \%$. 


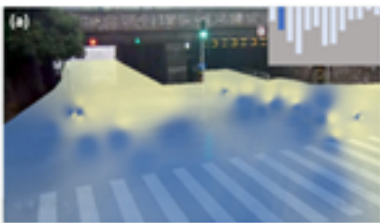

$16: 45$

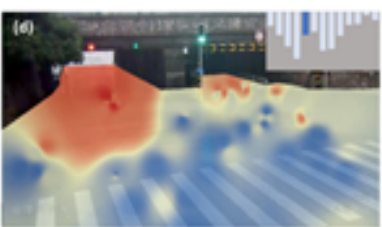

17:00

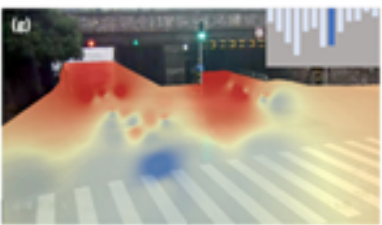

17:15

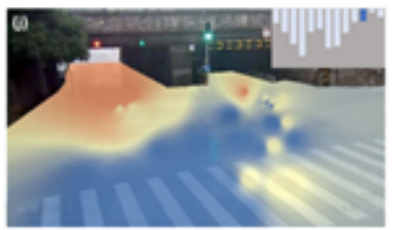

$17: 30$

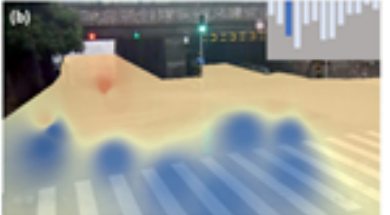

$16: 50$

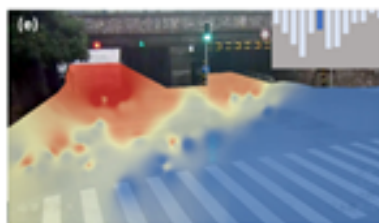

17:05

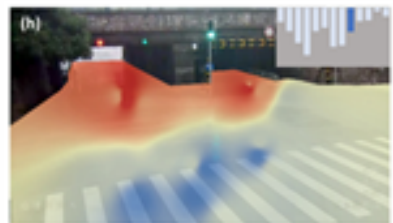

$17: 20$

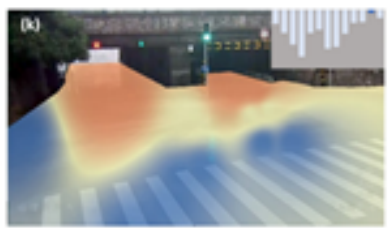

$17: 35$

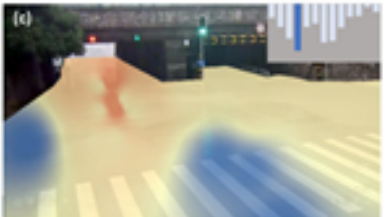

$16: 55$

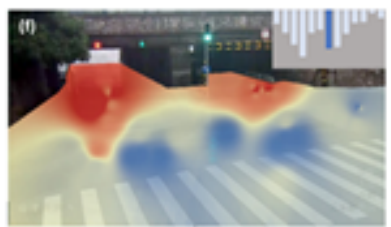

17:10

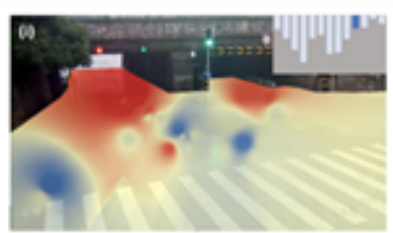

$17: 25$

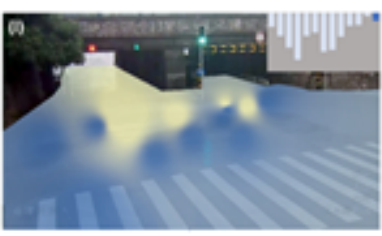

$17: 40$

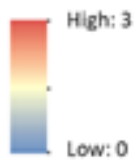

\section{Figure 8}

Estimated flood distribution map by data interpolation after deleting outliers of Case 1. The top of each group is the rainfall histogram in blue. Some areas in the scene have reached level 3 from 17:00, five minutes later than the peak of rainfall time series.

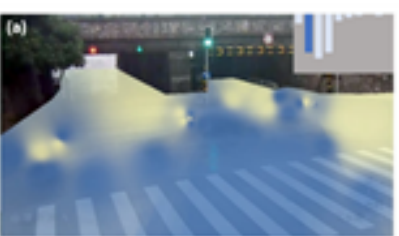

$15: 35$

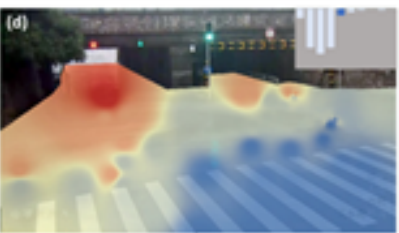

$15: 50$

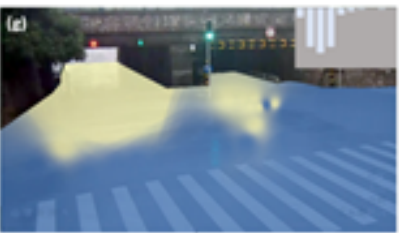

16:05

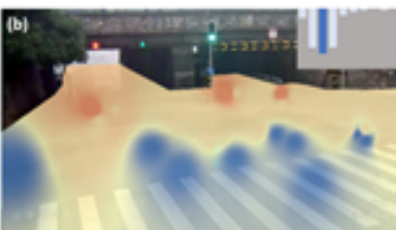

$15: 40$

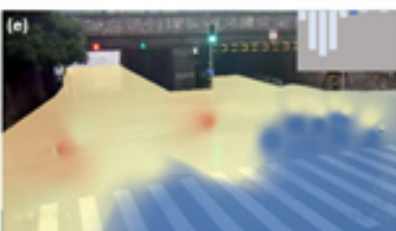

$15: 55$

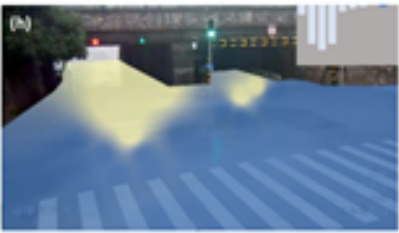

$16: 10$

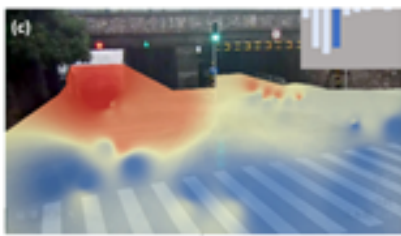

$15: 45$

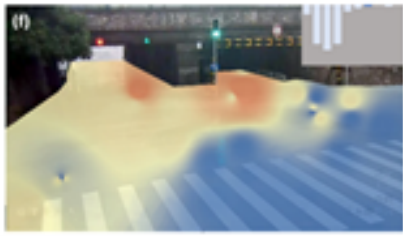

16:00

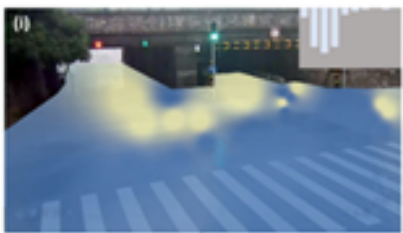

$16: 15$

Figure 9 
Estimated flood distribution map by data interpolation after deleting outliers of Case 2. The top of each group is the rainfall histogram in blue. Some areas in the scene have reached level 3 at 15:45, five minutes later than the peak of rainfall time series.
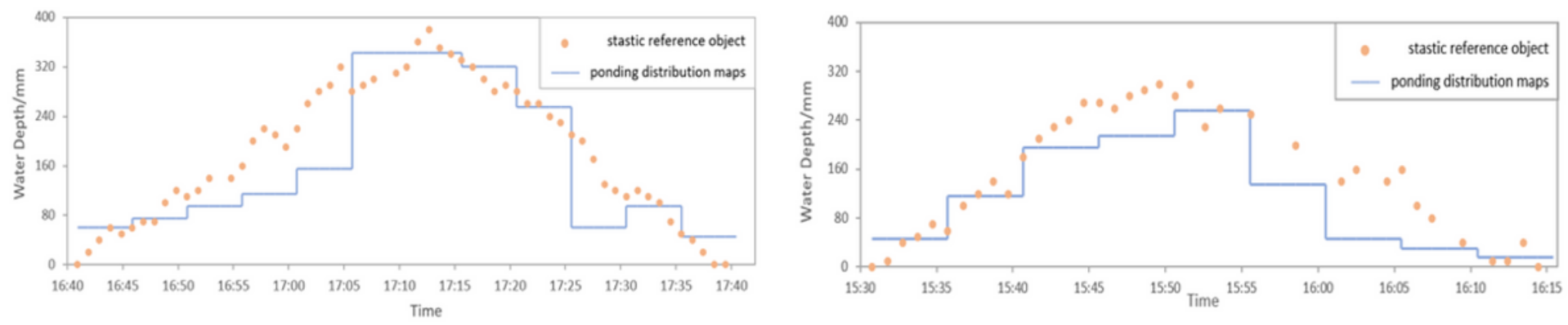

Figure 10

The comparison between water depth converted by ponding level extracted from ponding distribution maps (step line in blue) and the detected water depth time series based on static reference object (scatter in orange) for case 1 (left) and case 2 (right). The maximum water depth estimated by two methods appears at simultaneously, 17:10-17:15 in Case 1 and 15:50-15:50 in Case 2.

(a) (b) 
Four wrongly classified images caused by different reasons: (a) high beams; (b)obstacles in front of the predicted object; (c) vehicle angle; and (d) water splash. 